Altorientalische

Forschungen

Zeitschrift für den gesamten Orient im Altertum und frühen Mittelalter

Chefredakteur: HORST KLENGEL

Redaktionskollegium: ADELHEID BURKHARDT (Stellvertreter)

HELMUT FREYDANK

MARIA SCHETELICH

WERNER SUNDERMANN

THOMAS THILO

SIMONE RASCHMANN

INGRID WARNKE (Sekretär)

PETER ZIEME

Akademie Verlag 
Gedruckt mit Unterstützung der Deutschen Forschungsgemeinschaft

Bezugsmöglichkeiten

Bitte richten Sie Ihre Bestellungen an

VCH, Postfach 101161, D-W-6940 Weinheim, Telefon: (06201) 606152, Telefax: (06201) 606184, Telex: 465516 vchwh d.

Unsere Schweizer Kunden wenden sich bitte an

VCH, Hardstr. 10, CH-4020 Basel, Telefon: (061) 2710606, Telefax: (061) 2710618, Telex: $911527 \mathrm{dms}$ ch.

For our American customers

Altorientalische Forschungen (ISSN 0232-8461) is published by Akademie Verlag GmbH, Leipziger Str. 3-4, P. O. Box 1233, D-O-1086 Berlin, Germany and distributed by VCH Publishers, Inc., 303 N. W. 12th Ave., Deerfield Beach, FL 33442-1788 in North and South America; telefax (305) 428-8201; telephone (305) 428-5566 or 1-(800)-367-8249. Annual subscription price for two issues is U.S. $\$ 129,00$ including postage and handling. Application to Mail at Second-Class Postage Rates is pending at Boca Raton, FL 33431. U.S. POSTMASTER: Send address changes to VCH Publishers, Inc., 303 N.W. 12th Ave., Deerfield Beach, FL 33442-1788.

\section{Altorientalische Forschungen}

Herausgeber: Im Auftrag der Akademie Verlag GmbH herausgegeben von Prof. Dr. Horst Klengel.

Chefredakteur: Prof. Dr. Horst Klengel.

Anschrift der Redaktion: Altorientalische Forschungen, Unter den Linden 8, D - O-1080 Berlin.

Verlag: Akademie Verlag GmbH, Leipziger Str. 3-4, Postfach 1233, D-O-1086 Berlin; Telefon: 2236350 oder 2236351; Telefax: 2236357 oder 2236387; Bank: Dresdner Bank Weinheim, Konto-Nr. 754624 500, BLZ 67080050.

Geschäftsführer: Dr. Gerd Giesler, Dr. Bernhard Tesche.

Anzeigenannahme: Abt. Marketing/Produktinformation, Telefon: 2236365.

Gesamtherstellung: Druckerei „G. W. Leibniz“ GmbH, D-O-4450 Gräfenhainichen.

Erscheinungsweise: Die Zeitschrift erscheint jährlich in einem Band mit 2 Heften. Jahresbezugspreis 1992 Inland DM 180,- ; Ausland DM 187,- jeweils inklusive Versandkosten. Einzelheft DM 104,-.

Das Abonnement verlängert sich jeweils um ein weiteres Jahr, falls es nicht drei Monate vor Ablauf gekündigt wird.

Urhebertecht: Alle Rechte vorbehalten, insbesondere die der Übersetzungen. Kein Teil dieser Zeitschrift darf in irgendeiner Form - durch Photokopie, Mikrofilm oder irgendein anderes Verfahren - ohne schriftliche Genehmigung des Verlages reproduziert oder in eine von Maschinen, insbesondere von Datenverarbeitungsanlagen verwendbare Sprache übertragen oder übersetzt werden. - All rights reserved (including those of translations into foreign languages). No part of this issue may be reproduced in any form, by photoprint, microfilm or any other means, nor transmitted or translated into a machine language, without written permission from the publishers.

(C) 1992 by Akademie Verlag GmbH. Printed in the Federal Republic of Germany.

Der Akademie Verlag ist ein Unternehmen der VCH Verlagsgesellschaft. 
Die Autoren dieses Heftes der "Altorientalischen Forschungen" widmen ibre Beiträge ibrem Kollegen und langjäbrigen Bereichsleiter Prof. Dr. babil. Horst Klengel aus Anlaß seines 60. Geburtstages am 6. Januar 1993. 
